\title{
Calligraphy Therapy Interventions for Managing Depression in Cancer Patients: A Scoping Study
}

\section{Stephanie Wagner*}

Department of Complementary Medicine, Laurinsteig 16, 13465 Berlin, Germany

\begin{abstract}
For the last three decades, scientists have conducted research for medical therapy interventions of Chinese calligraphy handwriting $(\mathrm{CCH})$. Clinical research has found $\mathrm{CCH}$ has positive effects on behavioral and psychosomatic disorders: Depressive symptoms in cancer patients, psychiatric and cognitive disorders in elderly people, stress reduction, hyperarousal symptoms after earthquake, changes in theta waves and other diseases such as hypertension and Attention Deficit Hyperactivity.

Depression and anxiety are common among people diagnosed with cancer. $\mathrm{CCH}$ as a medical intervention was tested in Nasopharyngeal cancer patients (stage II and III). CCH improved certain stress symptoms and mood disturbances.

All studies showed a notable paucity for $\mathrm{CCH}$ intervention in medical therapy. However, to apply $\mathrm{CCH}$ for improving depressive symptoms in cancer patients and to strengthen the evidence, further research is required.
\end{abstract}

Keywords: Arts therapy; Cancer; Depression; Calligraphy; Calligraphy therapy

\section{Introduction}

This scoping study addresses the following research question: What is known from the literature about Chinese calligraphy handwriting $(\mathrm{CCH})$ interventions for managing depressive symptoms in cancer patients and $\mathrm{CCH}$ interventions used as medical therapy in general.

\section{Methods}

Electronic databases were searched to identify published studies investigating calligraphic interventions for managing depression in cancer patients and used as medical therapy in general. The searching strategies proposed by Arksey and O'Malley were as follows: 1 . identifying the research question; 2. identifying the relevant literature; 3 . study selection; 4 . charting the data and 5 . Collating the results $[1,2]$.

\section{Search Strategy}

The following electronic databases were searched: PubMed, Web of Science, ERIC, PsycINFO and Cochrane. The searches were conducted using the following keywords: calligraphy, calligraphy therapy, cancer and depression. The searches were districted to abstracts and articles published in English.

\section{Study Selection}

All identified abstracts and/or articles were screened by the following selection criteria: published in peer reviewed journals in full manuscript format, written in English language, published within the last ten years (2007 to 2017) and included calligraphic intervention which was compared to control group without intervention. Studies which met inclusion criteria were read in full. Data was extracted and summarized in Table 1 and Table 2 with the following design: author, country and year; intervention; participant characteristics; intervention design and details; assessment phases and outcome measures; results; type of calligraphy and limitations.

\section{Results}

Only one RCT (randomized controlled trial) tested a calligraphic intervention for managing depressive symptoms in cancer patients (Nasopharyngeal cancer with stage II and III) (Table 1) [3]. This study compared the effects of $\mathrm{CCH}$ with those of progressive muscle relaxation and imagery training on physiological arousal parameters, symptom distress and mood disturbance.

$\mathrm{CCH}$ demonstrated slow-down effects in heart rate, blood pressure and respiration rate. It also improved certain stress symptoms and mood disturbance.

Five RCTs were found for $\mathrm{CCH}$ interventions used as medical therapy in general (Table 2). In one RCT CCH therapy was used as part of multiple training modalities [4]. This study is not listed in Table 2 as $\mathrm{CCH}$ was not used as single intervention. Another study tested $\mathrm{CCH}$ to reduce stress symptoms in adults compared to meditation [5]. $\mathrm{CCH}$ decreased heart rate and increased skin temperature.

A fourth study showed that $\mathrm{CCH}$ can improve cognitive health in older people [6]. CCH improved orientation, attention and calculation in $\mathrm{CCH}$ group.

A fifth study investigated $\mathrm{CCH}$ therapy in children on hyperarousal symptoms after 2008 China earthquakes [7]. Arousal symptoms and salivary cortisol levels decreased in the intervention group. Last study provided evidence of cortical excitation in EEG theta waves coming from changes in the practitioner's body, emotions and cognition [8].

${ }^{*}$ Corresponding author: Stephanie Wagner, Department of Complementary Medicine, Laurinsteig 16, 13465-Berlin, Germany, Tel: +491776478545; E-mail: stephanie.wagner.sw@gmail.com

Received February 19, 2018; Accepted February 22, 2018; Published March 01, 2018

Citation: Wagner S (2018) Calligraphy Therapy Interventions for Managing Depression in Cancer Patients: A Scoping Study. Altern Integ Med 7: 260. doi: 10.4172/2327-5162.1000260

Copyright: ( 2018 Wagner S. This is an open-access article distributed under the terms of the Creative Commons Attribution License, which permits unrestricted use, distribution, and reproduction in any medium, provided the original author and source are credited. 
Citation: Wagner S (2018) Calligraphy Therapy Interventions for Managing Depression in Cancer Patients: A Scoping Study. Altern Integ Med 7: 260. doi: 10.4172/2327-5162.1000260

Page 2 of 3

\begin{tabular}{|c|c|c|c|c|c|c|c|}
\hline $\begin{array}{l}\text { Author, } \\
\text { country } \\
\text { and year }\end{array}$ & Intervention & $\begin{array}{l}\text { Participant } \\
\text { characteristics }\end{array}$ & $\begin{array}{l}\text { Intervention } \\
\text { design and details }\end{array}$ & $\begin{array}{l}\text { Assessment phases and } \\
\text { outcome measures }\end{array}$ & Results & $\begin{array}{l}\text { Type of } \\
\text { calligraphy }\end{array}$ & Limitations \\
\hline $\begin{array}{l}\text { Yang et } \\
\text { al. [3] }\end{array}$ & $\begin{array}{l}\mathrm{CCH} \text { and } \\
\text { relaxation } \\
\text { in cancer } \\
\text { patients }\end{array}$ & $\begin{array}{l}\text { - Nasopharyngeal } \\
\text { cancer (NPC) stage } \\
\text { II }(45.6 \%) \text { and III } \\
(54.4 \%) \\
\cdot \mathrm{N}=287, \\
\text { refusal rate } \mathrm{N}=197 \\
\cdot \mathrm{N}=90 \\
-\mathrm{N}=30 \text { relaxation } \\
\text { group, } \\
\text { dropouts }(\mathrm{n}=4) \\
\cdot \mathrm{N}=30 \mathrm{CCH} \text { group, } \\
\text { dropouts }(\mathrm{n}=6) \\
\cdot \mathrm{N}=30 \text { control } \\
\text { group, } \\
\text { dropouts }(\mathrm{n}=1) \\
\cdot \text { Mean age: } 49.63 \pm \\
10.81 \text { yrs. } \\
\text { (range: } 22-71 \text { yrs.) }\end{array}$ & $\begin{array}{l} \\
\text { - RCT } \\
\text { - Relaxation training } \\
30 \text { min/day for } 4 \\
\text { weeks (20 min } \\
\text { progressive muscle } \\
\text { relaxation, PMR) } \\
\text { followed by } 10 \text { min } \\
\text { guided imagery, GI) } \\
\text { - CCH-30 min/day } \\
\text { for } 4 \text { weeks } \\
\text { - no treatment for } \\
\text { control group }\end{array}$ & $\begin{array}{l}\text { - Ax phases: } \\
\text { T1-baseline } \\
\text { T2-2 weeks from T1 } \\
\text { T3-4 weeks from T1 } \\
\text { T4-FU, } 2 \text { weeks from T3 } \\
\text { - Physiological arousal } \\
\text { parameters } \\
\text { - heart rate, blood pressure, } \\
\text { respiration rate } \\
\text { - pre-post treatment } \\
\text { measurements on } 5 \text { days a } \\
\text { week from T1 to FU } \\
\text { - Psychosocial parameters: } \\
\text { - SDS (Symptom Distress } \\
\text { Scale) plus } 5 \text { added items } \\
\text { - Profile of Mood State-Short } \\
\text { Form (POMS-SF, Chinese } \\
\text { version) } \\
\text { - Karnofsky Performance } \\
\text { Status (KPS) }\end{array}$ & $\begin{array}{l}\text { - } \mathrm{N}=79 \text { in final analyses }(87.8 \%) \\
\text { - sig. lower heart rate, blood } \\
\text { pressure and repiration rate in two } \\
\text { intervention groups } \\
\text { - SDS } \\
\text { - sig. improved insomnia in relaxation } \\
\text { group at T2, T3 and FU } \\
\text { - sig. improved concentration in CCH } \\
\text { group at T2 and T3 } \\
\text { - KPS: no sig. treatment effect } \\
\text {-POMS-SF } \\
\text { - sig. improved tension-anxiety at T3 } \\
\text { and FU, depression injection at T2 } \\
\text { and T3, anger-hostility at T3 and FU } \\
\text { in relaxation group } \\
\text { - sig. improved depression injection } \\
\text { at T2 and T3, anger-hostility at T3 } \\
\text { and FU, fatigue-inertia at T2 and T3 } \\
\text { in CCH group }\end{array}$ & $\begin{array}{l}\text { Chinese } \\
\text { calligraphy } \\
\text { characters } \\
\text { from } \\
\text { handbook }\end{array}$ & $\begin{array}{l}\text { - high refusal } \\
\text { rate }\end{array}$ \\
\hline
\end{tabular}

Table 1: Studies - calligraphy therapy in cancer patients.

\begin{tabular}{|c|c|c|c|c|c|c|c|}
\hline $\begin{array}{l}\text { Author, } \\
\text { country } \\
\text { and year }\end{array}$ & Intervention & $\begin{array}{l}\text { Participant } \\
\text { characteristics }\end{array}$ & $\begin{array}{l}\text { Intervention } \\
\text { design and } \\
\text { details }\end{array}$ & $\begin{array}{l}\text { Assessment phases and } \\
\text { outcome measures }\end{array}$ & Results & $\begin{array}{l}\text { Type of } \\
\text { calligraphy }\end{array}$ & Limitations \\
\hline $\begin{array}{l}\text { Kao HSR } \\
\text { et al. [5] }\end{array}$ & $\begin{array}{l}\mathrm{CCH} \text { and } \\
\text { meditation for } \\
\text { stress reduction }\end{array}$ & $\begin{array}{l}\text { - } \mathrm{N}=30, \text { screened } \\
\text { by General Health } \\
\text { Questionnaire }(\text { Chinese } \\
\text { version of } \mathrm{GHQ}-28) \\
\cdot \mathrm{N}=10 \mathrm{CCH} \text { group } \\
\cdot \mathrm{N}=10 \text { meditation group } \\
\cdot \mathrm{N}=10 \text { control group } \\
\text { - age } 19-35 \text { yrs. }\end{array}$ & $\begin{array}{l}\text { - RCT } \\
\text { - one session/week } \\
\text { for } 8 \text { weeks } \\
\text { - treatment time } 33 \\
\text { min } 26 \mathrm{sec} \\
\text { - color test for } \\
\text { stress arousal } \\
\text { - standard } \\
\text { technique for } \\
\text { meditation } \\
\text { - no treatment for } \\
\text { control group }\end{array}$ & $\begin{array}{l}\text { - Ax phases: } \\
\text { before, during and after } \\
\text { each treatment session } \\
\text { - heart rate } \\
\text { - electromyographic scores } \\
\text { - skin temperature } \\
\text { - respiratory rate }\end{array}$ & $\begin{array}{l}\text { - sig. lower heart rate in } \mathrm{CCH} \\
\text { and meditation group } \\
\text { - sig. decrease in } \\
\text { electromyographic scores in } \\
\text { meditation and control group } \\
\text { - sig. increased skin } \\
\text { temperature in CCH and } \\
\text { meditation group } \\
\text { - sig. decreased respiratory } \\
\text { rate in meditation group }\end{array}$ & $\begin{array}{l}\text { Chinese } \\
\text { calligraphy } \\
\text { characters } \\
\text { from } \\
\text { handbook }\end{array}$ & $\begin{array}{l}\text { - small sample } \\
\text { size }\end{array}$ \\
\hline $\begin{array}{l}\text { Kwok et } \\
\text { al. [6] }\end{array}$ & $\begin{array}{l}\mathrm{CCH} \text { in cognitive } \\
\text { health }\end{array}$ & $\begin{array}{l}-\mathrm{N}=31 \text {, inclusion criteria } \\
\text { Chinese version of } \\
\text { the Mini-Mental State } \\
\text { Examination (CMMSE) } \\
\text { score } 20-25 \text {, mild cognitive } \\
\text { impairment } \\
\cdot \text { age }>70 \text { yrs. } \\
\cdot \mathrm{N}=14 \mathrm{CCH} \text { group } \\
\cdot \mathrm{N}=17 \text { control group }\end{array}$ & $\begin{array}{l}\text { - RCT } \\
\text { - } \mathrm{CCH} \text { for } 30 \mathrm{~min} \text {, } \\
\text { once a day, } 5 \\
\text { days/week, } \\
\text { for } 8 \text { weeks } \\
\text { - no treatment for } \\
\text { control group }\end{array}$ & $\begin{array}{l}\text { Ax phases: } \\
\text { - T1-baseline } \\
\text { - T2-8 weeks from T1 }\end{array}$ & $\begin{array}{l}\text { - CMMSE } \\
\text { - sig. improved orientation, } \\
\text { attention and calculation in } \\
\mathrm{CCH} \text { group } \\
\text { - sig. decline in orientation in } \\
\text { control group }\end{array}$ & $\begin{array}{l}\text { Chinese } \\
\text { calligraphy } \\
\text { characters } \\
\text { from } \\
\text { handbook }\end{array}$ & $\begin{array}{l}\text { - small sample } \\
\text { size } \\
\text { - only one } \\
\text { outcome } \\
\text { measure- } \\
\text { ment, CMMSE }\end{array}$ \\
\hline $\begin{array}{l}\text { Zhu et al. } \\
\text { [8] }\end{array}$ & $\begin{array}{l}\mathrm{CCH} \text { on } \\
\text { hyperaroual } \\
\text { symptoms } \\
\text { connected to } \\
\text { 2008 Sichuan } \\
\text { earthquakes in } \\
\text { China }\end{array}$ & $\begin{array}{l}\text { Experiment } 1 \\
\text { - } \mathrm{N}=210 \text {, selected from two } \\
\text { villages close to Sichuan } \\
\text { earthquake areas, one year } \\
\text { after disaster } \\
\text { - } \mathrm{N}=129 \mathrm{CCH} \text { group, age } \\
10.51 \pm 1.15 \text { yrs. } \\
\text { - } \mathrm{N}=81 \text { control group, age } \\
10.52 \pm 1.13 \text { yrs. } \\
\text { Experiment } 2 \\
\text { - } \mathrm{N}=41 \mathrm{CCH} \text { group, age } \\
10.52 \pm 1.16 \text { yrs. } \\
\text { - } \mathrm{N}=39 \text { control group, age } \\
10.54 \pm 1.15 \text { yrs. } \\
\text { - selected from earthquake } \\
\text { regions }\end{array}$ & $\begin{array}{l}\text { - RCT } \\
\cdot \text { CCH for } 1 \mathrm{hr} / \text { day, } \\
5 \text { days/week for } \\
30 \text { days } \\
\text { - control group } \\
\text { followed their } \\
\text { normal daily } \\
\text { routine }\end{array}$ & $\begin{array}{l}\text { Experiment } 1 \\
\text { - Ax Phases: } \\
\text { - T1- baseline } \\
\text { - T2-15 days from T1 } \\
\text { - T3-30 days from T1 } \\
\text { - Chinese version of } \\
\text { Children's Revised Impact } \\
\text { of Event Scale (CRIES-13) } \\
\text { Experiment } 2 \\
\text { - CRIES-13 at T1, T2 } \\
\text { and T3 } \\
\text { - salivary cortisol } \\
\text { measurement } \\
\text { - saliva sample taken on } \\
\text { T1, T2 and T3 } \\
\text { - cotton ball under tongue } \\
\text { for } \\
5 \text { min } \\
\text { - } 2 \text { ml sample } \\
\text { - centrifugation }\end{array}$ & $\begin{array}{l}\text { Experiment } 1 \\
\text { - sig. improved arousal } \\
\text { scores (CRIES-13) at T3 in } \\
\text { CCH group } \\
\text { Experiment } 2 \\
\text { - sig. lower salivary cortisol } \\
\text { in CCH group } \\
\text { - no information about } \\
\text { CRIES-13 scores }\end{array}$ & $\begin{array}{l}\text { Chinese } \\
\text { calligraphy } \\
\text { characters } \\
\text { from } \\
\text { handbook }\end{array}$ & $\begin{array}{l}\text { - no CRIES-13 } \\
\text { sore results in } \\
\text { experiment } 2\end{array}$ \\
\hline
\end{tabular}


Citation: Wagner S (2018) Calligraphy Therapy Interventions for Managing Depression in Cancer Patients: A Scoping Study. Altern Integ Med 7: 260. doi: 10.4172/2327-5162.1000260

Page 3 of 3

\begin{tabular}{|c|c|c|c|c|c|c|c|}
\hline $\begin{array}{l}\text { Xu, et } \\
\text { al.[7] }\end{array}$ & $\begin{array}{l}\mathrm{CCH} \text { on brain } \\
\text { function: cortical } \\
\text { excitation of theta } \\
\text { rhythm }\end{array}$ & $\begin{array}{l}-\mathrm{N}=16 \\
-\mathrm{N}=8 \mathrm{CCH} \text { group } \\
-\mathrm{N}=8 \text { control group }\end{array}$ & $\begin{array}{l}\text { - RCT } \\
-\mathrm{CCH} \text { training for } \\
10 \text { days, } 20 \mathrm{~min} / 10 \\
\text { sessions } \\
\text { - no treatment for } \\
\text { control group }\end{array}$ & $\begin{array}{l}\text { - Ax Phases: } \\
\text { - T1-baseline } \\
\text { - T2-10 days from T1 } \\
\text { - EEG waves (alpha and } \\
\text { theta waves) recordings } \\
\text { under four conditions: } \\
\text { eyes open, color test, } \\
\text { eyes closed, writing/sitting } \\
\text { quietly }\end{array}$ & $\begin{array}{l}\text { - sig. increased theta waves } \\
\text { in CCH group } \\
\text { - no sig. increased theta } \\
\text { waves in control group } \\
\text { - global style characters } \\
\text { evoked greater theta and } \\
\text { alpha waves than detailed } \\
\text { characters }\end{array}$ & $\begin{array}{l}\text { Characters } \\
\text { varying } \\
\text { in visual- } \\
\text { spatial } \\
\text { properties } \\
\text { (linear, } \\
\text { nonlinear, } \\
\text { global, } \\
\text { detailed) }\end{array}$ & $\begin{array}{l}\text { - one channel } \\
\text { EEG instad of } \\
\text { multi- } \\
\text { channels } \\
\text { - small character } \\
\text { number to } \\
\text { investigate } \\
\text { defined character } \\
\text { properties }\end{array}$ \\
\hline
\end{tabular}

Table 2: Studies - Overview about calligraphy therapy research.

\section{Conclusions}

There is a notable paucity of $\mathrm{CCH}$ interventions in medical therapy. Further research is required to strengthen the evidence base for $\mathrm{CCH}$ interventions in managing depression in cancer patients.

\section{References}

1. Brien SE, Lorenzetti DL, Lewis S, Kennedy J, Ghali WA (2010) Overview of a formal scoping review on health system report cards. Implement Sci 5:2.

2. Levac D, Colguhoun H, O'Brein KK (2010) Scoping studies: Advancing the methodology. Implement Sci 5: 69.

3. Yang XL, Li HH, Hong MH, Kao HS (2009) The effects of Chinese calligraphy handwriting and relaxation training in Chinese Nasopharyngeal Carcinoma patients: A randomized controlled trial. Int J Nurs Stud 47: 550-559.
4. Tai SY, Hsu CL, Huang SW, Ma TC, Hsieh WC, et al. (2016) Effects of multiple training modalities in patients with Alzhiemer's disease: A pilot study. Neuropsychiatr Dis Treat 12: 2843-2849.

5. Kao HSR, Zhu L, Chao AA, Chen HY, Liu ICY, et al. (2014) Calligraphy and meditation for stress reduction: An experimental comparison. Psychol Res Behav Manag 7: 47-52.

6. Kwok TC, Bai X, Kao HS, Li JC, Ho FK (2011) Cognitive effects of calligraphy therapy for older people: a randomized controlled trial in Hong Kong. Clin Interv Aging 6: 269-273.

7. Xiu M, Kao HSR, Zhang M, Lam SPW, Wang W (2013) Cognitive-Neural effects of brush writing of Chinese characters: Cortical excitation of theta rhythm. Evid Based Complement Alternat Med 975190.

8. Zhu Z, Wang R, Kao HS, Zong Y, Liu Z, et al. (2014) Effect of calligraphy training on hyperarousal symptoms for childhood survivors of the 2008 China earthquakes. Neuropsychiatr Dis Treat 10: 977-985. 